\title{
Satisfacción vital y su relación con otras variables psicosociales en población española residente en Alemania
}

\section{Life satisfaction and its connection with other psycho-social variables in Spanish residents in Germany}

\author{
Macarena Vallejo-Martín y María del Pilar Moreno Jiménez \\ Universidad de Málaga, España
}

Disponible online 31 de agosto de 2016

\begin{abstract}
Este estudio analiza qué variables psicosociales están relacionadas con la satisfacción vital en población española residente en Alemania. Para ello se consideran las siguientes: apoyo social, autoestima, satisfacción laboral y apego e identidad con el lugar. Se tienen en cuenta también factores sociodemográficos, en concreto: empleo, conocimiento del alemán, ingresos mensuales recibidos y clase social percibida. Con una muestra de 206 personas, los resultados muestran un nivel medio o medio-alto en las variables psicosociales consideradas y correlaciones significativas entre ellas. Respecto a los factores sociodemográficos, no se muestran diferencias significativas en satisfacción vital por el hecho de tener empleo, pero sí para el resto de factores en sentido ascendente. Entre ellos, aquel de mayor efecto es la clase social percibida. Los resultados también indican que satisfacción laboral intrínseca, clase social percibida, apego al lugar y autoestima son variables predictivas de satisfacción vital, explicando un $55.5 \%$ de la varianza. Según lo anterior, se observan diferencias con otros estudios sobre satisfacción vital en población inmigrante.
\end{abstract}

Palabras Clave: Emigración Española; Satisfacción Vital; Satisfacción Laboral Intrínseca; Clase Social Percibida.

This study analysed the psychosocial variables related to life satisfaction in a population of Spanish residents in Germany. The psychosocial variables were as follows: social support, self-esteem, job satisfaction, and attachment to and identification with the place of residence. Sociodemographic factors were also considered: job position, level of ability in the German language, monthly income, and perceived social class. The sample comprised 206 participants. The results showed that the participants had medium or medium-high scores on all the psychosocial variables and that there were significant correlations between them.Regarding the sociodemographic factors, although no significant association was found between life satisfaction and having a job, significant associations were found between life satisfaction and the other factors. Of these factors, perceived social class had the biggest effect. The results also showed that intrinsic job satisfaction, perceived social class, attachment to the place of residence, and self-esteem predicted life satisfaction (explaining $55.5 \%$ of variance). These results are in contrast with other studies on life satisfaction in immigrant populations.

Key Words: Spanish Immigration; Life Satisfaction; Intrinsic Job Satisfaction; Perceived Social Class.

Correspondencia: Macarena Vallejo-Martín. Facultad de Psicología, Campus de Teatinos, Universidad de Málaga, Fax: 952131100, email: mvallejo@uma.es. E-mail de la co-autora: Pilar Moreno Jiménez: mpilar@uma.es 
La realidad migratoria en el sur de Europa está cambiando de tendencia en los últimos años. Aunque se sigue recibiendo población migrada de otras zonas geográficas, la crisis socioeconómica ha hecho que el flujo migratorio se invierta. Este hecho se constata para los países denominados PIGS (de su denominación en inglés: Portugal, Italia, Grecia y España). Los países de destino principales son aquellos situados al Norte de Europa. El estudio de esta nueva realidad migratoria debe ser, por su elevada relevancia social, una prioridad para los científicos sociales y para la sociedad europea en su conjunto.

Igualmente, en la actualidad en España cada vez son más las personas que toman la decisión de emigrar a otros países buscando mejores condiciones de vida. Así, se invierte la dirección del flujo migratorio respecto a las últimas décadas, siendo desde comienzos de 2011 mayor el número de personas que abandonan España que el de las que llegan (INE, 2012). Entre los principales países de destino se encuentran Reino Unido, Francia y Alemania. González-Ferrer elevaba ya en 2013 a 700.000 la cifra de españoles que habían emigrado con la crisis económica, frente a los datos oficiales que estimaban en 225.000 los nacionales que se habían marchado entre 2008 y 2012. El informe se basa en la comparación de datos de inmigración de los países de destino respecto a los que ofrecen fuentes españolas, que trasmiten una información sesgada y parcial de la realidad migratoria. En el año 2014, según el INE, han emigrado a Alemania un total de 5.733 españoles (INE, 2014).

Más allá del baile de cifras, los psicólogos debemos preguntarnos qué variables psicosociales determinan el bienestar subjetivo de esta población en el nuevo contexto. Desde el surgimiento de la Psicología Positiva, la disciplina psicosocial ha acrecentado su interés por el estudio de los factores relacionados con las dimensiones psicológicas del bienestar (Neto, 1995). Le subyace la convicción de que el bienestar es sensible a la influencia de variables psicosociales, especialmente en grupos minoritarios como es la población migrante.

El bienestar subjetivo consta de dos componentes: la cognición y el afecto (Veenhoven, 1984; 1991). El componente cognitivo, denominado satisfacción vital, hace referencia a un juicio global sobre la propia vida, es decir, una valoración que se realiza a largo plazo sobre si las condiciones de vida responden a las aspiraciones. Referido a población inmigrante, corresponde a la valoración que hace el sujeto de su propia experiencia migratoria en relación a que su vida se desarrolle de acuerdo a metas, creencias, valores y deseos propios (Marrero \& Carballeira, 2010). Numerosos autores establecen niveles bajos de bienestar para este colectivo comparados con la población no migrante, que sugieren una influencia negativa del hecho migratorio (Bilbao, Techio, Basabe \& Páez, 2007; Hernández, Pozo \& Alonso, 2004; Vallejo-Martín, 2009; Zoblina \& Páez, 2004). Interesa en este estudio qué variables se relacionan con una evaluación positiva o discrepancia entre aspiraciones y logros de la población emigrante española. El rango puede oscilar entre la sensación de realización personal y las de fracaso y frustración. Para ello, se parte de las variables especificadas por los modelos más relevantes en la literatura respecto a los procesos migratorios, a saber: Modelo de Aculturación de Berry (Berry, Kim, Power, Young \& Bujaki, 1989); Modelo de Scott y Scott (1989); Modelo de Parker y McEvoy (1993); Modelo Interactivo de Aculturación (Bourhis, Möise, Perreault \& Senécal, 1997); Modelo Ecológico Contextual de Aculturación de Birman (1998); Modelo de Piontkowski, Florack, Hoelker y Obdrzálek (2004); y en España el Modelo Ampliado de Aculturación Relativa (Navas et. al, 2005) y el Modelo de Satisfacción Vital para Inmigrantes (Vallejo-Martín \& Moreno-Jiménez, 2014).

En líneas generales, la migración se produce como una forma de mejorar las condiciones de vida que en la actualidad se traduce, en último término, en encontrar un trabajo donde la persona pueda desarrollarse de manera óptima. Ello comporta una evaluación subjetiva que le corresponde al sujeto, pudiendo ir desde el hecho mismo de tener un empleo a que éste se dé en unas condiciones económicas favorables o estar acorde a las expectativas, cualificación y formación. En cualquier caso, dependerá de las necesidades mínimas que estén cubiertas en cada caso. Dicha evaluación subjetiva está relacionada con la satisfacción laboral. Locke (1976) la concibe como un estado emocional positivo o placentero de las experiencias laborales del trabajador. Las características del propio trabajo y las de cada trabajador condicionarán esa respuesta afectiva. Warr, Cook y Wall (1979) diferenciaron entre satisfacción laboral extrínseca e intrínseca. La primera se centra en la satisfacción del trabajador con aspectos relativos a la organización del trabajo, como la jornada laboral, la remuneración o las condiciones físicas del mismo. La satisfacción laboral intrínseca aborda aspectos como el reconocimiento obtenido por el trabajo, responsabilidad, promoción y aspectos relativos al contenido de la tarea. Según Martínez (1996), la inserción laboral de las personas inmigrantes se produce en el mercado de trabajo precario, desprotegido e informal. Aun así, se torna un elemento central en el proceso migratorio. La satisfacción laboral y su relación con la satisfacción con la vida en este colectivo dependerán no sólo de tener un trabajo, sino también de la calidad del mismo en relación a que las compensaciones psico-socio-económicas estén acordes a las expectativas (Vallejo-Martín \& MorenoJiménez, 2012).

En relación a la satisfacción con la vida, otra de las variables determinantes es el apoyo social. De hecho, ha ocupado un lugar central como factor social determinante de la salud y el bienestar psicológico (Cobb, 1976; Cohen, Gottlieb \& Underwood, 2002; Eckersley, Dixon \& Douglas, 2001; Gracia, 1997; Montero, 2003; Ramírez \& Cusmille, 1997). De esta forma, si los recursos de apoyo son importantes mediadores entre los eventos vitales estresantes y la salud y el bienestar, 
en el caso de los inmigrantes esta relación adquiere especial relevancia (Aroian, 1992; Cohen \& Wills, 1985; Delgado \& Humm-Delgado, 1982; Golding \& Baezconde-Garbanati, 1990; Martínez, García, Maya, Rodríguez \& Checa, 1996; Salvador, Pozo \& Alonso, 2010). Así lo confirman diferentes estudios en nuestro país en los que se destaca que la red social es básica para promover el éxito del nuevo proyecto social de la persona desplazada (Martínez, 1996; Martínez, García \& Maya, 1999; 2002, Vallejo-Martín \& Moreno-Jiménez, 2014).

Por otro lado, la autoestima también ha sido estudiada con frecuencia como expresión psicológica del bienestar subjetivo (Branscombe, Schmitt \& Harvey, 1999; Crocker \& Major, 1989). De hecho, se la considera uno de los mayores predictores de la satisfacción con la vida (Kang, Shaver, Sue, Ming \& Jing, 2003; Lewinsohn, Redner \& Seeley, 1991; MartínezAntón, Buelga \& Cava, 2007). En el nuevo contexto y para el desarrollo del proyecto migratorio, la valoración que la persona hace de sí misma, así como de su endogrupo, será fundamental en relación a la evaluación de la consecución de metas y aspiraciones.

Por último, se destacan dos variables psicoambientales en el presente estudio: apego e identidad con el lugar. Estas dos variables han recibido poca atención en los modelos de aculturación. Sin embargo, cómo las personas migrantes se relacionan con su nuevo lugar de residencia, tanto individual como colectivamente, se cree de suma importancia en el estudio de la satisfacción vital. El apego al lugar hace referencia al vínculo afectivo entre las personas y determinados lugares, aquellos sentimientos positivos sobre lo que uno posee y los factores de tipo emocional ante los lugares (Jorgensen \& Steadman, 2001). Para Hidalgo y Hernández (2001) el apego al lugar es el vínculo que las personas establecen con sitios específicos, en los que prefieren permanecer y en los que se encuentran confortables y seguros. La identidad con el lugar se trata de un componente de la identidad personal, en función de los elementos que caracterizan un determinado lugar y de la naturaleza de las interacciones que en él acontecen (Wester-Herber, 2004). En relación con el hecho migratorio, en un estudio realizado en nuestro país se encuentran puntuaciones superiores tanto en apego como en identidad con el lugar en autóctonos respecto a los inmigrantes (Hernández, Hidalgo, Salazar \& Hess, 2007). Esto puede ser debido a que cuando un individuo procede de otro lugar debe pasar por un proceso de aculturación que implica el aprendizaje de algunos aspectos (significados y valores) y la socialización individual y grupal (Fan, 1998).
El objetivo de este estudio es analizar la satisfacción vital de población española residente en Alemania y su relación con diferentes variables psicosociales. Según los estudios mencionados anteriormente, se eligen las siguientes: satisfacción laboral (intrínseca y extrínseca), apoyo social, autoestima y apego e identidad con el lugar. Además, se estudiará la satisfacción vital en relación a tener empleo, la clase social percibida, los ingresos mensuales y el conocimiento de alemán. En función de estos objetivos, y según lo señalado en la literatura referida a población inmigrante y satisfacción vital, particularmente el Modelo de Aculturación de Berry (Berry et al., 1989) y el Modelo de Satisfacción vital para Inmigrantes (Vallejo-Martín \& Moreno-Jiménez, 2014) en los que dichas variables psicosociales y factores sociodemográficos juegan un papel central, se plantean las siguientes hipótesis:

1. La satisfacción vital correlacionará en sentido positivo y significativamente con cada una de las variables psicosociales consideradas.

2. Los niveles de satisfacción vital serán mayores por el hecho de tener un empleo, una mejor percepción de la clase social, mayores ingresos mensuales y mejor conocimiento del alemán.

3. Las variables psicosociales (apego e identidad con el lugar, satisfacción laboral extrínseca e intrínseca, apoyo social y autoestima) y factores sociodemográficos tomados en consideración (clase social, ingresos mensuales y conocimiento del alemán) predecirán en sentido positivo la satisfacción vital.

\section{Participantes}

\section{Método}

La muestra del estudio está compuesta de 206 personas españolas que han emigrado a Alemania, de las cuales el 65\% son mujeres y el $35 \%$ son hombres. La media de edad se sitúa en los 30 años y el $85 \%$ dice no tener hijos. El periodo medio de residencia es de 21 meses.

La mayoría de las personas encuestadas son trabajadores activos, encontrándose una tasa de empleo del $88 \%$. Respecto al nivel de estudios, el $73.7 \%$ poseen estudios universitarios. La motivación principal para emigrar ha sido la búsqueda de empleo, la falta de oportunidades en España y poder ejercer una profesión acorde a la cualificación. Casi la mitad de la muestra, el 48.8\%, manifiesta que seguirá residiendo en Alemania según las circunstancias laborales. A continuación, se describen otras características de la muestra:

Tabla 1

Características de la muestra según factores sociodemográficos.

\begin{tabular}{|c|c|c|c|c|c|c|c|}
\hline \multicolumn{2}{|l|}{ Clase social destino } & \multicolumn{2}{|c|}{ Clase social España } & \multicolumn{2}{|c|}{ Conocimiento alemán } & \multicolumn{2}{|l|}{ Ingresos mensuales } \\
\hline Baja/media-baja & $35.9 \%$ & Baja/media-baja & $22.9 \%$ & Bajo/medio-bajo & $29.4 \% *$ & Menos de 1200 euros & $29.9 \%$ \\
\hline Media & $49.5 \%$ & Media & $58.8 \%$ & Medio & $34.5 \%$ & Entre 1200 y 2500 & $53.4 \%$ \\
\hline Media-alta/alta & $14.6 \%$ & Media-alta/alta & $18.6 \%$ & Medio-alto/alto & $35.9 \%$ & Más de 2500 euros & $16.7 \%$ \\
\hline
\end{tabular}


Por otra parte, respecto a porcentajes de participación, estos difieren según el tipo. Así, los referidos a participación sociopolítica en Alemania son bajos, encontrándose un 3.9\% para la adscripción a un sindicato o partido político. Para una participación en un movimiento social (participación comunitaria) el porcentaje es del $12.6 \%$. Éste se incrementa hasta el $33.7 \%$ referido a participación sociopolítica en un grupo español en Alemania. El 12.2\% manifiesta acudir a asociaciones de ayuda a inmigrantes.

\section{Instrumentos}

Se les preguntó a los participantes sobre factores sociodemográficos tales como la edad, sexo, clase social percibida, nivel de estudios, tiempo de residencia en Alemania, ingresos mensuales y conocimiento del alemán. A continuación, se describen los diferentes instrumentos utilizados para cada una de las variables psicosociales:

Escala de Satisfacción vital (Diener, Emmons, Larsen \& Griffin, 1985). Mide el grado de satisfacción que las personas tienen con su vida globalmente. Consta de cinco ítems, siendo un ejemplo: "Hasta ahora he obtenido las cosas importantes que quiero en mi vida". Las respuestas van en un rango de puntuación de 1 a 5. Los coeficientes de fiabilidad han alcanzado en este estudio un de .84 .

Escala de Apoyo social Duke-UNC (Broadhead, Gehlbach, Degruy \& Kaplan, 1988). Mide apoyo afectivo y confidencial. Está compuesta por un total de once ítems. Un ejemplo puede ser "Cuento con personas que se preocupan de lo que me sucede". La escala de respuesta tiene un rango de puntuaciones de 1 a 5. Se obtiene un Alfa de Cronbach para este estudio de .88 .

Escala de Autoestima (Rosenberg, 1965). Mide la valoración que la persona tiene de sí misma. La forman diez ítems, como por ejemplo "En general, estoy satisfecho conmigo mismo" El rango de respuesta oscila entre unas puntuaciones de 1 y 4 . El Alfa de Cronbach para este estudio es de .83.

Escala de Apego e identidad con el lugar (Hernández et al., 2007). Consta de dos subescalas. La primera mide el vínculo emocional hacia los vecinos y lugar donde se reside; la segunda, la identificación con el mismo. La subescala de apego al lugar la componen un total de ocho ítems, tales como "En esta ciudad me siento como en mi casa". La subescala de identidad con el lugar la conforman cuatro ítems, como por ejemplo: "Me siento identificado con esta ciudad". Ambas subescalas se contestan con un rango de puntuaciones de 1 a 5 . El Alfa de Cronbach para este estudio es .93 y .94 respectivamente.

Escala de Satisfacción laboral (Warr et al., 1979). Compuesta por dos subescalas: satisfacción laboral extrínseca y satisfacción laboral intrínseca. La primera está formada por ocho ítems y la segunda por siente. Como ejemplo de ítem de la subescala de satisfacción laboral extrínseca se puede señalar "Grado de satisfacción con su salario. Para la subescala de satisfacción laboral intrínseca un ejemplo puede ser: "Grado de satisfacción con las posibilidades de progreso y promoción".
Ambas subescalas presentan respuestas con un rango de puntaciones de 1 a 7 . El Alfa de Cronbach para este estudio es de .94 para la primera y de .92 para la segunda.

\section{Procedimiento}

Los datos fueron recogidos desde finales de 2013 hasta finales de 2014 en diferentes ciudades de Alemania, siendo las principales Múnich, Berlín y Frankfurt. Estas ciudades fueron seleccionadas por dos motivos: el elevado número de población española residente y disponer en ellas de los primeros contactos para la recogida de datos.

El cuestionario fue administrado en castellano. La participación de las personas encuestadas en el estudio era anónima y voluntaria. Se les indicaba que la investigación tenía un carácter exclusivamente académico y que los datos serían analizados en el conjunto de la muestra, no de forma individual.

Se realiza un muestreo no aleatorio utilizando la técnica bola de nieve (Taylor \& Bogdan, 1986), debido a la carencia de información con ausencia de datos estadísticos fiables y a la dispersión de la población. Se parten de diez contactos previos distribuidos en las diferentes ciudades, obteniendo un total de 206 personas con una reproducción desigual que oscila entre 0 y 3 nuevos entrevistados.

\section{Análisis estadístico}

En primer lugar, se comprobó la fiabilidad de las escalas para este estudio. Posteriormente se calculó la puntuación media para cada una de las variables y el coeficiente de correlación de Pearson. Seguidamente se estudiaron diferencias de medias intergrupos según distintos factores utilizando las pruebas $t$-Student (para sexo y empleo) y ANOVA (para clase social percibida, ingresos mensuales y conocimiento del alemán). En este segundo caso, una vez comprobado la existencia de diferencias significativas y homogeneidad de varianzas (Prueba Levene $p>.05$ en todos los casos) se realizaron pruebas post hoc-Tukey para detectar las diferencias intergrupos. También se llevó a cabo una prueba de contraste de tipo polinómico que permite obtener comparaciones de tendencias en solución ponderada y el tipo de relación de la variable dependiente con los otros factores.

Por último, se realizó un análisis de regresión múltiple para conocer en qué medida las variables y factores considerados predecían la satisfacción vital (método introducir). Se comprobó la no existencia de autocorrelación y colinealidad. Las variables categóricas (clase social percibida, ingresos mensuales y conocimiento del alemán) se codificaron como dummy tomando como referencia su nivel más bajo.

\section{Resultados \\ Niveles de las variables psicosociales y correlaciones}

En la siguiente tabla se recogen los niveles de cada una de las variables consideradas en el estudio y su coeficiente de correlación: 
Tabla 2

Medias, desviación típica y coeficientes de correlación de las variables estudiadas.

\begin{tabular}{|c|c|c|c|c|c|c|c|c|}
\hline & $M$ & $D T$ & SL intrínseca & SL extrínseca & Autoestima & Apego lugar & Identidad lugar & Apoyo social \\
\hline Satisfacción vital (1-5) & 2.94 & .92 & $.586 * *$ & $.515 * *$ & $.361 * *$ & $.474 * *$ & $.462 * *$ & $.483 * *$ \\
\hline S. L. Intrínseca (1-7) & 4.69 & 1.29 & & $.830 * *$ & $.254 * *$ & $.309 * *$ & $.253 * *$ & $.259 * *$ \\
\hline S. L. Extrínseca (1-7) & 4.94 & 1.29 & & & $.225 * *$ & $.233 * *$ & $.171 *$ & $268 * *$ \\
\hline Autoestima (1-4) & 3.34 & .51 & & & & $.166^{*}$ & $.174 *$ & $.357 * *$ \\
\hline Apego al lugar (1-5) & 3.42 & 1.29 & & & & & $.846 * *$ & $.496 * *$ \\
\hline Identidad lugar (1-5) & 2.89 & 1.48 & & & & & & $.495 * *$ \\
\hline Apoyo social (1-5) & 3.65 & .79 & & & & & & \\
\hline
\end{tabular}

Nota: SL intrínseca: Satisfacción laboral intrínseca; SL extrínseca: Satisfacción laboral extrínseca; ${ }^{*} p<.05 ; * * p<.01$.

Tal y como puede observarse, las personas encuestadas muestran un nivel medio de satisfacción vital; es decir, por lo general se encuentran satisfechos con lo realizado hasta el momento y su vida responde en un término medio a sus aspiraciones. Asimismo, manifiestan un nivel alto de autoestima y de apoyo social, por lo que se valoran bien a sí mismos y se sienten valorados y queridos por los demás. El nivel de apego al lugar es medio-alto y medio para la identificación con el lugar. Por tanto, se encuentran a gusto en la ciudad en la que residen, aunque ésta no forme en gran medida parte de su identidad. Respecto a la satisfacción laboral, se obtiene un nivel medio-alto tanto para la satisfacción intrínseca como para la extrínseca, aunque los niveles son ligeramente inferiores en la segunda.

Por otra parte, todas las variables consideradas correlacionan significativamente entre ellas. En concreto, para satisfacción vital destaca la relación con satisfacción laboral, tanto intrínseca $(r=.586)$ como extrínseca $(r=.515)$, seguido del apoyo social $(r=.483)$. Por otro lado, también han de resaltarse otros coeficientes de correlación como, por un lado, los mostrados entre satisfacción laboral extrínseca e intrínseca $(r=.830)$ $\mathrm{y}$, por otro, entre apego e identidad con el lugar $(r=.846)$. Estos resultados son coherentes pues, en ambos casos, la literatura muestra estas variables estrechamente relacionadas.

\section{Diferencias en satisfacción vital según clase social perci- bida, ingresos mensuales y conocimiento del alemán}

Al aplicar $t$-Student, se observa que no se encuentran diferencias estadísticamente significativas entre grupos para el factor sexo $[t(188)=.112, p>.05, d=.02]$ ni tampoco para el factor empleo $[t(187)=.089, p>.05, d=.02]$.

Para la comparación intergrupos en el resto de factores: clase social percibida, ingresos mensuales y conocimiento del alemán, se aplica la prueba ANOVA. Los resultados indican diferencias significativas para clase social percibida $[F(2,187)$ $\left.=40.27, p<.001, \eta_{\text {parcial }}^{2}=.224\right]$; ingresos mensuales $[F(2$, $\left.186)=8.83, p<.001, \eta_{\text {parcial }}^{2}=.012\right]$ y conocimiento del alemán $\left[F(2,187)=7.89, p<.001, \eta_{\text {parcial }}^{2}=.033\right]$. Los índices obtenidos a través de Eta cuadrado parcial señalan que las diferencias halladas entre las distintas clases sociales deben ser tomadas en consideración.

Se lleva a cabo un análisis post hoc de Tukey para detectar entre qué grupos aparecen las diferencias en los tres factores. Los resultados se muestran en la siguiente tabla:
Tabla 3

Diferencias en satisfacción vital según clase social, ingresos y conocimiento del alemán $(N=206)$.

\begin{tabular}{llllll}
\hline Clase social $^{1}$ & $M$ & Ingresos mensuales $^{2}$ & $M$ & Conocimiento alemán $^{3}$ & $M$ \\
\hline (a) Baja/media-baja & 2.32 & Menos de $1200 €$ & 2.72 & Bajo/medio-bajo & 2.73 \\
(b) Media & 3.24 & Entre 1200 y $2500 €$ & 2.88 & Medio & 2.77 \\
(c) Media-alta/alta & 3.72 & Más de $2500 €$ & 3.58 & Medio/alto & 3.32 \\
\hline
\end{tabular}

Nota: 1 = Tukey, las diferencias son significativas para un nivel .05 . Se encuentran diferencias en clase social entre (a) y (b); (a) y (c); (b) y (c); 2 = Tukey, las diferencias son significativas para un nivel .01. Se encuentran diferencias en ingresos mensuales entre (a) y (c); (b) y (c); 3 $=$ Tukey, las diferencias son significativas para un nivel .01 . Se encuentran diferencias en clase social entre (a) y (c); (b) y (c).

Se realiza una prueba de contraste polinómico que permite obtener comparaciones de tendencias en solución ponderada. En todos los casos la relación de cada uno de los factores con la variable satisfacción vital es lineal (Clase social $[F(1,187)$ $=77.34, p<.000]$; Ingresos mensuales $[F(1,186)=13.8, p<$ $.000]$; Conocimiento del alemán $[F(1,187)=12.43, p<.005])$. A continuación se muestran en la Figura 1:

Figura 1

Comparaciones de tendencias en satisfacción vital según clase social percibida, ingresos mensuales y conocimiento del alemán.

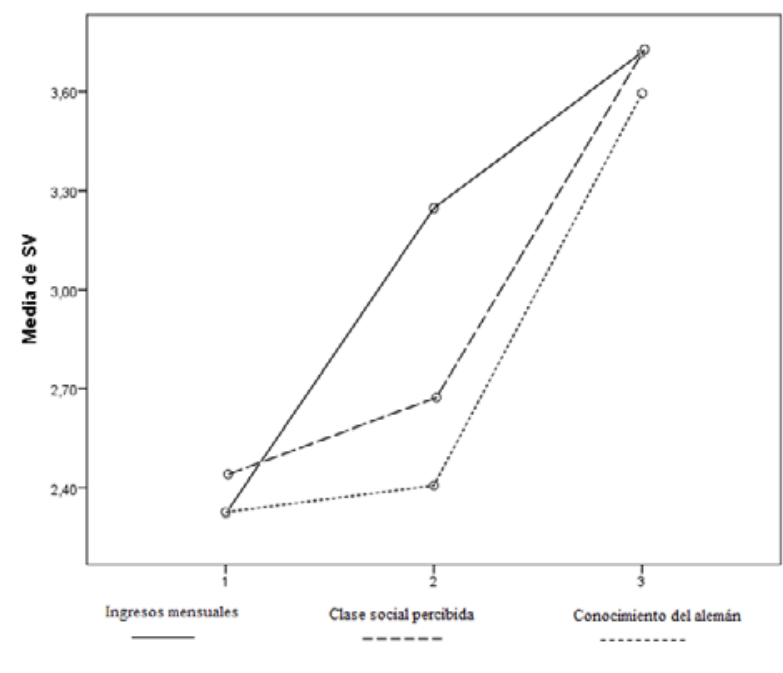

\section{Variables predictivas para la satisfacción vital}

Para conocer qué variables predicen la satisfacción vital se realiza un análisis de regresión introduciendo todas las variables: autoestima, apoyo social, apego e identidad con el 
lugar, satisfacción laboral intrínseca y extrínseca, clase social percibida, ingresos mensuales y conocimiento del alemán. Las tres últimas, al ser variables categóricas, se codificaron como dummy tomando como referencia su nivel más bajo. Los resultados se recogen en la tabla 4 .

Tabla 4

Análisis de Regresión múltiple para satisfacción vital.

\begin{tabular}{lrrrrr}
\hline & $\beta$ & $\mathrm{B}$ & $P$ & $F$ & $\mathrm{R}^{2}$ cor. \\
\hline S. L. intrínseca & .128 & .206 & .002 & $47.47 * * *$ & .520 \\
S. L. extrínseca & .065 & .091 & .172 & & \\
Apoyo Social & .056 & .046 & .198 & & \\
Autoestima & .345 & .185 & .000 & & \\
Apego lugar & .185 & .243 & .000 & & \\
Identidad lugar & .003 & .005 & .935 & & \\
Clase social baja-media & .349 & .178 & .000 & & \\
Clase social media-alta & .724 & .253 & .000 & & \\
Ingresos bajos-medios & .113 & .057 & .157 & & \\
Ingresos medios-altos & .269 & .109 & .013 & & \\
Idioma bajo-medio & .029 & .014 & .757 & & \\
Idioma medio-alto & .107 & .055 & .242 & & \\
\hline
\end{tabular}

Nota: $R^{2}$ cor.: Coeficiente de determinación corregido; $* * * p<.001$.

Según se puede observar, las variables consideradas explican el $52 \%$ de la varianza, siendo estadísticamente significativas para la predicción de la satisfacción vital las siguientes: satisfacción laboral intrínseca, autoestima, apego al lugar, diferencias en clases social percibida en todos sus niveles e ingresos mensuales en sus niveles más altos.

\section{Discusión}

Los flujos migratorios son un fenómeno a escala mundial que tiene importantes repercusiones tanto a nivel individual como grupal (comunidad de origen y comunidad de destino). En los últimos años, consecuencia de la crisis socioeconómica, los países sureuropeos se han convertido no sólo en países receptores de flujos migratorios, sino también, en países emisores. Este es el caso de España, en el que un número considerable de ciudadanos han emigrado a otros países de Europa. Esta nueva realidad ha de ser estudiada en profundidad. No sólo por la responsabilidad social de la sociedad científica para favorecer el desarrollo de estos procesos migratorios de manera óptima, sino también, como forma de alumbrar pistas a una verdadera política de integración global. El presente trabajo trata de acercarse a este fenómeno poniendo el foco de interés en conocer las variables psicosociales relacionadas con cómo las personas emigradas valoran globalmente su vida. Para ello se elige uno de los principales países de destino de la población emigrante española: Alemania.

La muestra participante en este estudio pertenece a una población joven cualificada, con una media de edad de 30 años, en la que casi dos tercios tienen estudios universitarios. La motivación principal para emigrar ha sido la búsqueda de empleo y ejercer una profesión acorde al nivel de formación. Más de la mitad se percibe como clase media. También alrededor de la mitad de la muestra recibe como ingresos entre los 1200 y 2500 euros al mes y condiciona su permanencia en el país a circunstancias laborales. Por tanto, se puede determinar que se trata de una emigración preparada y con formación, para la que juega un papel central un empleo de calidad.

El conjunto de la muestra presenta niveles altos en autoestima. Los resultados sobre la alta valoración personal de sí mismo coincide con los hallazgos en algunos estudios respecto a población inmigrante (Murillo \& Molero, 2012; Vallejo-Martín \& Moreno-Jiménez, 2014). Por otro lado, se han encontrado niveles medios-altos en al apego al lugar, al contrario de lo que aparece en otros estudios con niveles más bajos (Casakin, Ruiz \& Hernández, 2013; Hernández et al., 2007; Ríos \& MorenoJiménez, 2010, 2012). Los españoles en Alemania sienten un vínculo afectivo fuerte con los vecinos de su comunidad. Igualmente, los niveles son superiores a los esperados en apoyo social. También se han hallado niveles medios-altos en satisfacción laboral intrínseca y extrínseca. Otros estudios sobre satisfacción laboral en nuestro país para población inmigrante muestran niveles inferiores (Moreno-Jiménez, Ríos, Canto, San Martín \& Perles, 2010). Por último, se han encontrado niveles medios en identidad con el lugar y satisfacción vital. Por tanto, los españoles en Alemania no sienten una fuerte identificación con este país y hacen un juicio global medio sobre su vida.

Todas las variables mencionadas anteriormente correlacionan de forma positiva y significativa con satisfacción vital, cumpliéndose la hipótesis 1 . En concreto, ha de resaltarse unos niveles altos de correlación con la satisfacción laboral, tanto intrínseca como extrínseca. Por lo tanto, a medida que las personas se encuentren más a gusto y recompensadas en su trabajo mayor será la creencia de que la vida responde a las expectativas y aspiraciones, y viceversa.

Respecto a las diferencias entre grupos en satisfacción vital según clase social, ingresos mensuales, conocimiento del alemán y empleo, puede decirse que se cumple parcialmente la hipótesis 2. Llama la atención que no se encuentren diferencias por el hecho de tener un empleo. El trabajo proporciona, o debería proporcionar, salud física y mental, prestigio, entretenimiento, contacto social y realización personal (Gamero, 2009; Salanova, Gracia \& Peiró, 1996, entre otros). Sin embargo, las personas desplazadas, al insertarse en el mercado laboral, lo hacen en general en trabajos temporales que son rechazados por otros y mal pagados, siendo condiciones laborales negativas especialmente el salario, el tipo de trabajo, la temporalidad, etc. (Solé, 2001). Esto puede determinar que el hecho en sí mismo de tener un empleo no sea suficiente para que las personas hagan un juicio global positivo de su vida. Sin embargo, sí parece tener un efecto ascendente un buen conocimiento del alemán, unos ingresos altos y percibirse en una clase social alta. De hecho, en este último factor se muestran diferencias significativas para los tres grupos (clase social baja/media-baja, clase media y clase social media-alta/alta) y los resultados indican tener un efecto importante en relación a la satisfacción vital. Por tanto, podemos dilucidar que en el juicio global que este colectivo hace de su vida es importante la percepción del estatus social y económico propio. 
Por otra parte, los resultados del análisis de regresión indican que las variables que se muestran como significativas para la predicción de la satisfacción con la vida son: satisfacción laboral intrínseca, apego al lugar, autoestima, clase social percibida (en todos sus niveles) e ingresos mensuales en sus niveles más altos; por tanto, se cumple parcialmente la hipótesis 3. Todas ellas predicen en un 52\% la satisfacción vital. De esta forma, para que las personas juzguen que su vida responde a sus aspiraciones es importante ser reconocido en el trabajo, tener cierta autonomía, capacidad de promoción, etc. También percibirse en una clase social media o alta y contar con unos ingresos mensuales elevados, tener vínculos afectivos con la comunidad en la que se reside y tener un buen concepto de sí mismo. Según los resultados obtenidos en este estudio, para la predicción de la valoración global que los españoles emigrantes en Alemania hacen de su vida, no es especialmente relevante el grado de conocimiento del alemán, la satisfacción extrínseca que les reporta su trabajo o el apoyo percibido de sus amigos y familiares.

En líneas generales, los resultados encontrados ponen de manifiesto que, para la población española emigrante en Alemania, es de suma importancia la calidad y tipo de trabajo que se ejerce. Quizás este hecho esté determinado porque la mayoría tiene un empleo, adquiriendo más relevancia otros factores como que éste se adecue al nivel de formación, se realice en unas condiciones óptimas y genere unos ingresos que proporcionen una buena posición económica. Ello resulta congruente con aquellos trabajos que han insinuado una motivación económica de los procesos migratorios (Boneva \& Frieze, 2001; Murillo, 2009). También con aquellos estudios que han señalado que mayores ingresos tiende a corresponderse con mayores niveles de bienestar y satisfacción con la vida (DeVoe \& Pfeffer, 2009; Kahn \& Juster, 2002).

Por otro lado, los resultados obtenidos en este estudio difieren de lo resaltado en otros referidos a población inmigrante en España (Vallejo-Martín \& Moreno-Jiménez, 2014). Estas diferencias pueden deberse a que, en este último colectivo, el hecho de no tener un empleo repercute asimismo en la situación administrativa, con lo que puede suponer de riesgo de expulsión, reclusión en centros de internamiento, difícil acceso a servicios públicos, etc. Sin embargo, para los emigrantes españoles, aunque puedan darse algunos problemas burocráticos como el acceso a una vivienda, el hecho de no tener un empleo no supone la pérdida del reconocimiento de ciudadanía. Así, el ser ciudadano comunitario puede mediatizar la percepción como inmigrante en el nuevo lugar de residencia. Este hecho puede determinar que uno de los factores con más peso en la predicción de la satisfacción con la vida sea la satisfacción laboral intrínseca. Es decir, sentirse valorado en el trabajo, que éste se adecue al nivel de formación, que permita desarrollarse y hacer uso de las competencias y habilidades, etc. Tal y como reflejan algunos estudios sobre migraciones intraeuropeas éstas están movidas, además de por una motivación económica y de movilidad social, por otras como el reconocimiento social o la búsqueda de una calidad de vida mayor (Ackers, 1998, 2005; Ackers, Bryony \& Jess, 2007; Iredale, 1999, 2001; Mahroum, 1999, 2001; Pellegrino, 2001; Salt, 1992, 1997).

Dicho lo anterior, sin embargo, ha de decirse que una de las limitaciones de este estudio es que casi tres tercios de la muestra se corresponden a personas universitarias. Esto puede suponer un sesgo en cómo se percibe el empleo y la calidad del mismo. Aunque todo parece indicar que la población española que emigra es una población cualificada, no hay un seguimiento riguroso de este colectivo por parte de las instituciones públicas, tanto en número como en características. Además, la ausencia de estudios previos referidos a población española emigrada en los años recientes hace difícil su comparación. Por otra parte, también sería interesante conocer si la satisfacción vital de este colectivo se ve influenciada por el tiempo de residencia en el nuevo país, dado que en su mayoría se trata de una nueva ola de emigración aún no asentada en la sociedad de acogida.

Los psicólogos sociales debemos seguir estudiando este fenómeno como forma de contribuir al desarrollo psicosocial de la población que se ha visto abocada a emigrar. De esta forma, a tenor de los resultados, sería importante estudiar en futuras investigaciones si la población española desplazada se siente "inmigrante" en otros países de Europa y si percibe prejuicios por parte de la población autóctona. En cualquier caso, tal y como señalan Perkins, Palmer y García-Ramírez (2011), la investigación sobre migrantes debe responder a las necesidades de la comunidad y estar orientada a la acción y al cambio social positivo.

\section{Referencias}

1. Ackers, L. (1998). Shifting Spaces: Women, Citizenship and Migration Within the European Union. Bristol: The Policy Press.

2. Ackers, L. (2005). Moving People and Knowledge: Scientific Mobility in the European Union. International Migration, 43, 99-131. http://dx.doi.org/10.1111/j.1468$\underline{2435.2005 .00343 . \mathrm{x}}$

3. Ackers, L., Bryony G. \& Jess, G. (2007). Moving People and Knowledge: Scientific Mobility in an Enlarging European Union. A summary Report, University of Liverpool. http://dx.doi.org/10.4337/9781848444867

4. Aroian, K. J. (1992): Sources of social support and conflict for Polish immigrants. Qualitative Health Research, 2, 178207. http://dx.doi.org/10.1177/104973239200200205

5. Berry, J.W., Kim, U., Power, S., Young, M. \& Bujaki, M. (1989). Acculturation attitudes in plural societies. Applied Psychology, 38, 185-206. http://dx.doi. org/10.1111/j.1464-0597.1989.tb01208.x

6. Bilbao, M. A., Techio, E., Basabe, N. \& Paéz, D. (2007). Intergración social y bienestar subjetivo y felicidad en los jóvenes: una comparación entre autóctonos e inmigrantes. En Memorias II European Community Psychology Associa- 
tion Seminar, 46-47. Universidad de Sevilla, Sevilla.

7. Birman, D. (1998). Biculturalism and perceived competence of Latino immigrant adolescents. American Journal of Community Psychology, 26, 335-354. http://dx.doi. org/10.1023/A:1022101219563

8. Branscombe, N. R., Schmitt, M. T. \& Harvey, R. D. (1999). Perceived group and personal discrimination: differential effects on personal self-esteem. European Jorunal of Social Psychology, 36, 773-789.

9. Boneva, B. \& Frieze, I. H. (2001). Toward a concept of a migrant personality. Journal of Social Issues, 57, 477-491. http://dx.doi.org/10.1111/0022-4537.00224

10. Bourhis, R., Moïse, L.C., Perreault, S. \& Senecal, S. (1997). Towards an interactive acculturation model: A social psychological approach. International Journal of Psychology, 32, 369-386. http://dx.doi.org/10.1080/002075997400629

11. Broadhead, W. E., Gehlbach, S. H., Degruy, F. V. \& Kaplan, B. H. (1988). The Duke-UNC functional social support questionnaire: measurement of social support in family medicine patients. Med Care, 26, 709-723. http://dx.doi. org/10.1097/00005650-198807000-00006

12. Casakin, H., Ruiz, C. \& Hernández, B. (2013). Diferencias en el desarrollo del apego y la identidad con el lugar en residentes no nativos de ciudades de Israel y ciudades de Tenerife. Estudios de Psicología, 34, 287-297. http:// dx.doi.org/10.1174/021093913808349325

13. Cobb, S. (1976). Social support as a moderator of life stress. Psychosomatic Medicine, 38, 300-314. http://dx.doi. org/10.1097/00006842-197609000-00003

14. Cohen, S., Gottlieb, B. H. \& Underwood, L. G. (2002). Social relationships and health. En S. Cohen, L. G. Underwood \& B. H. Gottlieb (Eds.), Social support measurement and intervention: A guide for health and social scientists (pp. 3-25). Oxford: Oxford University Press. http://dx.doi. org/10.1093/med:psych/9780195126709.003.0001

15. Cohen, S. \& Wills, T. A. (1985). Stress, social support and the buffering hypothesis. Psychological Bulletin, 98, 310357. http://dx.doi.org/10.1037/0033-2909.98.2.310

16. Crocker, J. \& Major, B. (1989). Social stigma and selfesteem: The self-prtotective properties os stigma. Psychological Review, 96, 608-630. http://dx.doi.org/10.1037/0033295X.96.4.608

17. Delgado, M. \& Humm-Delgado, D. (1982): Natural support systems: source of strength in Hispanic communities. Social Work, 27, 83-89.

18. DeVoe, S. \& Pfeffer, J. (2009). When is happiness about how much you earn? The effect of hourly payment on the money-happiness connection. Personality and Social Psychology Bulletin, 35, 1602-1618. http://dx.doi. org/10.2139/ssrn.1420893

19. Diener, E., Emmons, R.A., Larsen, R.J. \& Griffin, S. (1985). The Satisfaction with Life Scale. Journal of Personality Assessment, 49, 71-75. http://dx.doi.org/10.1207/ s15327752jpa4901_13

20. Eckersley, R., Dixon, J. \& Douglas, B. (eds.) (2001). The social origins of health and well-being. Nueva York: Cambridge University Press.

21. Fan, C. N. (1998). Canada as a new place: the immigrant's experience. Journal of Environmental Psychology 18, 55-67. http://dx.doi.org/10.1006/jevp.1997.0065

22. Gamero, C. (2009). Valoración de las características de los empleos por nativos e inmigrantes: el enfoque de la satisfacción con la vida. Principios. Estudios de Economía Politica, 14, 93-111.

23. Golding, M. J. \& Baezconde-Garbanati, L. A. (1990). Ethnicity, Culture and Social Resources. American Journal of Community Psychology, 18, 359-370. http://dx.doi. org/10.1007/bf00938118

24. González-Ferrer, A. (2013). La nueva emigración española. Lo que sabemos y lo que no. Zoom Político. Madrid, Laboratorio de Alternativas.

25. Gracia, E. (1997). El apoyo social en la intervención comunitaria. Barcelona: Paidós.

26. Hernández, B., Hidalgo, M.C., Salazar, M.E. \& Hess. S. (2007). Place attachment and place identity in natives and non natives. Journal of Environmental Psychology, 27, 310- 319. http://dx.doi.org/10.1016/j.jenvp.2007.06.003

27. Hernández, S., Pozo, C. \& Alonso, E. (2004). Apoyo social y bienestar subjetivo en un colectivo de inmigrantes: ¿efectos directos o amortiguadores? Boletín de Psicología, 80, 79-96.

28. Hidalgo, M. C. \& Hernández, B. (2001). Place Attachment: conceptual and empirical questions. Journal of Environmental Psychology, 21, 273-281. http://dx.doi.org/10.1006/ jevp.2001.0221

29. INE (2012). Estadística de migraciones 2012. Instituto Nacional de Estadística., Gobierno de España. Recuperado de http://www.ine.es/prensa/np788.pdf

30. INE (2014). Flujo de emigración con destino al extranjero 2014. Instituto Nacional de Estadística Gobierno de España. Recuperado de http:/www.ine.es/jaxi/tabla.do?path=/t20/ p277/prov/e01/10/\&file $=01010$. px \& type $=$ pcaxis $\& L=0$

31. Iredale, R. (1999). The Need to Import Skilled Personnel: Factors Favouring and Hindering its International Mobility. International Migration, 37, 89-123. http://dx.doi. org/10.1111/1468-2435.00067

32. Iredale, R. (2001). The Migration of Professionals: Theories and Typologies. International Migration, 39, 7-24. http://dx.doi.org/10.1111/1468-2435.00169

33. Jorgensen, B. S. \& Stedman, R. (2001). Sense of place as an attitude: lakeshore property owners' attitudes toward their properties. Journal of Environmental Psychology, 21, 233-248. http://dx.doi.org/10.1006/jevp.2001.0226

34. Kahn, R. \& Juster, F. T. (2002). Well-Being: Concepts and measures. Journal of Social Issues, 58, 627-644. http:// dx.doi.org/10.1111/1540-4560.00281 
35. Kang, S. M., Shaver, P., Sue, S., Ming, K. H. \& Jing, H. (2003). Culture-specific patterns in the prediction of life satisfaction: Roles of emotion, relationship quality, and selfesteem. Personality and Social Psychology Bulletin, 29, 1596-1608. http://dx.doi.org/10.1177/0146167203255986

36. Lewinsohn, P. M., Redner, E. \& Seeley, J. R. (1991). The relationship between life satisfaction and psychosocial variables: New perspectives. En F. Strack, M. Argyle \& N. Schwarsz (Eds.), Subjective well-being: Interdisciplinary perspective (pp. 193-212). Nueva York: Pergamon.

37. Locke, E. A. (1976). The nature and causes of job satisfaction. En M.D. Dunnette (Ed.), Handbook of Industrial and Organizational Psychology (pp. 1297-1349). Chicago, IL: Rand McNally.

38. Mahroum, S. (1999). Competing for the highly skilled: Europe in Perspective. Science and Public Policy, 26, 17-25. http://dx.doi.org/10.3152/147154399781782608

39. Mahroum, S. (2001). Europe and the Immigration of Highly Skilled Labour. International Migration, 39, 27-42. http:// dx.doi.org/10.1111/1468-2435.00170

40. Marrero, R. J. \& Carballeira, M. (2010). El papel del optimismo y del apoyo social en el bienestar subjetivo. Salud Mental, 33, 39-46.

41. Martínez, M. C. (1996). Análisis psicosocial del prejuicio. Madrid: Síntesis.

42. Martínez, M. F., García, M. \& Maya, I. (1999). El papel de los recursos sociales naturales en el proceso migratorio. Intervención Psicosocial, 8, 221-232.

43. Martínez, M. F., García, M. \& Maya, I. (2002). Social support and locus of control as predictors psychological well-being in Moroccan and Peruvian immigrant women in Spain. International Journal of Intercultural Relations, 26, 287-310. http://dx.doi.org/10.1016/S01471767(02)00005-6

44. Martínez, M. F., García, M., Maya, I, Rodríguez, S. \& Checa, F. (1996). La Integración Social de los Inmigrantes Africanos en Andalucía. Necesidades y Recursos. Sevilla: Junta de Andalucía.

45. Martínez-Antón, M., Buelga, S. \& Cava, M. J. (2007). La satisfacción con la vida en la adolescencia y su relación con el auto-estigma y el ajuste escolar. Anuario de Psicología, 38, 293-303.

46. Montero, M. (2003). Teoría y práctica de la psicología comunitaria: La tensión entre comunidad y sociedad. Barcelona: Paidós.

47. Moreno-Jiménez, M. P., Ríos, M. L. Canto, J. M., San Martín, J. \& Perles, F. (2010). Satisfacción laboral y Burnout en Trabajos poco cualificados: diferencias entre sexos en población inmigrante. Revista de Psicología del Trabajo y las Organizaciones, 26, 255-265. http://dx.doi. org/10.5093/tr2010v26n3a8

48. Murillo, J. (2009). Rostros de la migración. Bogotá: Fundación Esperanza-Unión Europea.
49. Murillo, J. \& Molero, F. (2012). La satisfacción vital: su relación con el prejuicio, la identidad nacional, la autoestima y el bienestar material en inmigrantes. Acta Colombiana de Psicología 15, 99-108.

50. Navas, M., García, M. C., Sánchez, J., Rojas, A. J., Pumares, P. \& Fernández, J. S. (2005). Relative Acculturation Extended Model (RAEM): New contributions with regard to the study of acculturation. International Journal of Intercultural Relations, 29, 21-37. http://dx.doi.org/10.1016/j. ijintrel.2005.04.001

51. Neto, F. (1995). Predictors of satisfaction with life among second generation migrants. Social Indicators Research, 35, 93-116. http://dx.doi.org/10.1007/BF01079240

52. Parker, B. \& McEvoy, G.M. (1993). Initial Examination of a Model of Intercultural Adjustment. International Journal of Intercultural Relations, 17, 355-379. http://dx.doi. org/10.1016/0147-1767(93)90039-B

53. Pellegrino, A. (2001). Trends in Latin American Skilled Migration: Brain Drain or Brain Exchange? International Migration, 39, 111-132. http://dx.doi.org/10.1111/14682435.00174

54. Perkins, D. D., Palmer, N. A. \& García-Ramírez, M (2011). Estudios psicosociales sobre migraciones y comunidad. Psychosocial Intervention, 20, 1-7.

55. Piontkowski, U. \& Florack, A., Hoelker, P. \& Obdrzálek, P. (2004). Predicting acculturation attitudes of dominant and non-dominants groups. International Journal of Intercultural Relations, 24, 1-26. http://dx.doi.org/10.1016/S01471767(99)00020-6

56. Ramírez, V. \& Cumsille, P. (1997). Evaluación de la eficiencia de un programa comunitario de apoyo a la maternidad adolescente. Revista Latinoamericana de Psicología, 29, 267-286.

57. Ríos, M. L. \& Moreno-Jiménez, M. P. (2010). Influencia de la participación comunitaria y la identidad con el lugar en la satisfacción vital en inmigrantes. Escritos de Psicología, 3, 8-16.

58. Ríos, M. L. \& Moreno-Jiménez, M. P. (2012). Place identity and residential satisfaction: differences between natives and immigrants. Psyecology, 3/1, 15-26. http://dx.doi. org/10.1174/217119712799240224

59. Rosenberg, M. (1965). La autoimagen del adolescente y la sociedad. Buenos Aires: Paidós (traducción de 1973).

60. Salanova, M., Gracia, F. J. \& Peiró, J. M. (1996). Significado del trabajo y valores laborales. En J. M. Peiró \& F. Prieto (Eds), Tratado de Psicología del Trabajo. Vol. II: Aspectos psicosociales del trabajo (pp.35-63). Madrid. Síntesis.

61. Salt, J. (1992). Migration Processes Among the Highly Skilled Europe. The International Migration Review, 26, 484505. http://dx.doi.org/10.2307/2547068

62. Salt, J. (1997). International Movements of the Highly Skilled. Paris: OECD. 
63. Salvador, M. C., Pozo, C. \& Alonso, E. (2010). Estrategias comportamentales aculturación y Síndrome de Ulises de los inmigrantes latinoamericanos. Boletín de Psicología, 98, 55-72.

64. Scott, W. \& Scott, R. (1989). Adaptation of immigrants: individual differences and determinants. International series in experimental social psychology, vol, 18. Oxford: Pergamon Press.

65. Solé, C. (2001). El impacto de la inmigración en la economía y en la sociedad receptora. Barcelona: Antrophos

66. Taylor, S.J. \& Bogdan, R. (1986). Introducción a los métodos cualitativos de investigación. Buenos Aires: Paidós

67. Vallejo-Martín, M. (2009). Satisfacción vital en el proceso de aculturación de inmigrantes: propuesta de un modelo. [Tesis Doctoral no publicada], Departamento de Psicología Social, Antropología Social, Trabajo Social y Servicios Sociales, Universidad de Málaga.

68. Vallejo-Martín, M. \& Moreno-Jiménez, M. P. (2012). An Evaluation of Life Satisfaction within the migratory experience according to psychosocial variables. Psychology, 3, 1248-1253. http://dx.doi.org/10.4236/psych.2012.312A185

69. Vallejo-Martín, M. \& Moreno-Jiménez, M. P. (2014). Del culturalismo al bienestar psicológico: propuesta de un modelo de satisfacción vital en el proceso de aculturación de Inmigrantes. Boletín de Psicología, 110, 53-67.
70. Veenhoven, R. (1984). Conditions of hapiness. Boston: Reidel. http://dx.doi.org/10.1007/978-94-009-6432-7

71. Veenhoven, R. (1991). Is happiness relative? Social Indicators Research, 24, 1-34. http://dx.doi.org/10.1007/ BF00292648

72. Warr, P., Cook, J. \& Wall, T. (1979). Scales for the measurement of some work attitudes and aspects of psychological well-being. Journal of Occupational Psychology, 52, 129-148. http://dx.doi.org/10.1111/j.2044-8325.1979. tb00448.x

73. Wester-Herber, M. (2004). Underlying concerns in landuse conflicts the role of place identity in risk perception. Environmental Science \& Policy, 7, 109-116. http://dx.doi. org/10.1016/j.envsci.2003.12.001

74. Zoblina, A. \& Páez, D. (2004). Choque cultural y percepción de las diferencias culturales en la población inmigrante en España. Encuentros en Psicología Social, 1, 210-216.

Fecha de recepción: 23 de julio, 2015 Fecha de recepción de la versión modificada: 20 de enero, 2016 Fecha de aceptación: 28 de marzo, 2016 